\title{
IMPLANTAÇÃO DE UMA FÁBRICA DE CERÂMICA VERMELHA: ESTUDO DE CASO PARA PEQUENA EMPRESA*
}

\author{
Léo Daiki Shinomiya ${ }^{1}$ \\ Andre Luiz Vilaça do Carmo ${ }^{2}$ \\ Joner Oliveira Alves ${ }^{3}$ \\ Raphael de Paiva Barbosa 4
}

\section{Resumo}

O trabalho apresenta estudos de cenários técnico e econômico para a implantação de uma pequena fábrica cerâmica, através do estudo de caso de uma prospecção de argila vermelha no interior do Pará. O estudo consistiu na execução de sondagem à trado (ST) na área, para cálculo volumétrico da reserva, e recolhimento de amostra para caracterizações em laboratório. A argila coletada foi submetida a análises do teor de umidade, química (Fluorescência de Raios-X) e mineralógica (Difratometria de Raios-X). Um cenário para uma empresa entre micro e pequena produtora de cerâmica foi estabelecido visando estimativas preliminares para um possível empreendimento. A análise de cenários levou a uma possibilidade de implementação de uma unidade fabril de cerâmica com capacidade produtiva de até 140 mil peças/mês. Restrições quanto ao tipo de produto desejado e a característica da argila prospectada devem ser consideradas, podendo ser necessário a mistura de argilas de base "gorda", com maior quantidade de argilominerais.

Palavras-chave: Avaliação de cenários; Indústria cerâmica; Argila vermelha; Pequena empresa.

\section{DEPLOYMENT OF A RED CERAMIC MILL: CASE STUDY FOR SMALL BUSINESS}

\begin{abstract}
The work presents studies of technical and economic scenarios for the deployment of a small ceramics mill, through the case study of a prospecting red clay in the Pará state. The study consisted of an auger drilling on the area for volumetric calculation of the reserve, and sample collection for laboratorial characterization. Clay collected was subjected to analyses of moisture content, chemical composition (X-ray fluorescence) and mineralogical characterization (X-ray diffraction). A scenario of a business among micro and small ceramic mill was established aiming preliminary estimates for a possible venture. The scenario analyses led to the implementing possibility of a ceramic plant with capacity of up to 140,000 pieces/month. Restrictions of the type of desired product and characteristics of the clay may be considered, due to possible requirements of a mixture of base clays "fat", with higher amounts of clay minerals.
\end{abstract}

Keywords: Scenarios assessment; Ceramic industry; Red clay; Small business.

1 Eng. Mecânico, Mestre em Eng. Mecânica e de Produção, Assistente de Pesquisa, Instituto SENAI de Inovação em Tecnologias Minerais, Federação das Indústrias do Estado do Pará, Belém - PA.

2 Químico Industrial, Mestre em Geologia e Geoquímica, Assistente de Pesquisa, Instituto SENAI de Inovação em Tecnologias Minerais, Federação das Indústrias do Estado do Pará, Belém - PA.

3 Bacharel em Física, Doutor em Eng. Metalúrgica e de Materiais, Diretor, Instituto SENAI de Inovação em Tecnologias Minerais, Federação das Indústrias do Estado do Pará, Belém - PA.

4 Bacharel em Ciências Econômicas, Doutor em Ciências Socioambientais, Gerente de Inovação e Projetos, Instituto SENAI de Inovação em Tecnologias Minerais, Federação das Indústrias do Estado do Pará, Belém - PA. 


\section{INTRODUÇÃO}

Argila é um material de ocorrência natural, formada predominantemente por minerais de granulometria fina, que, quando misturada a determinadas proporções de água, torna-se plástica e que endurece ao ser seca ou queimada [1]. Grande parte da argila lavrada no país é destinada às indústrias, tais como, energia, metalomecânica, cerâmica, entre outras. Segundo o Anuário Mineral Brasileiro de 2010 [2], existem várias aplicações para argilas e dentre estas destaca-se o setor da cerâmica vermelha. Tal segmento engloba produtos de minerais não metálicos da indústria de transformação, fornecendo produtos que servem como base para a construção civil, como blocos de vedação e estruturais, telhas, tijolos maciços, lajes, pisos, etc. [3, 4, 5]. A Tabela 1 apresenta o consumo de argila por setores da indústria.

Tabela 1. Distribuição setorial do consumo de argila [2]

\begin{tabular}{lc}
\hline \multicolumn{1}{c}{ Setor } & Consumo (\%) \\
\hline Cerâmica Vermelha & 33,52 \\
\hline Construção Civil & 21,68 \\
\hline Pisos e Revestimentos & 17,71 \\
\hline Cimento & 6,09 \\
\hline Aterro & 2,72 \\
\hline Cerâmica Branca & 2,09 \\
\hline Construção/Manutenção de estradas & 1,55 \\
\hline Artefatos de Cimento & 1,27 \\
\hline Outros & 13,37 \\
\hline
\end{tabular}

A indústria de cerâmica vermelha, por ser um setor diretamente ligado a construção civil, se encontra espalhada por todo o território nacional, tanto na forma de pequenas empresas de organização simples até pequenas olarias. São, geralmente, encontradas próximas as suas jazidas de matéria prima e aos mercados consumidores, devido ao seu produto de baixo valor agregado, o que inviabiliza seu transporte a longas distâncias. Esses aglomerados produtivos, são constituídos, tanto pelos núcleos cerâmicos como pelos mercados vizinhos e vem sendo denominados de Arranjos Produtivos Locais (APLs) [4, 5].

No estado do Pará, há dois APLs que são amplamente conhecidos. Estão localizados nas cidades de Santarém e São Miguel do Guamá (oeste e nordeste do estado, respectivamente) [4]. Entretanto, há outras localidades de menor expressão, tal como na cidade de Marabá, sudeste do Pará, a qual também apresenta uma considerável produção de cerâmica vermelha [6], bem como regiões em que esta atividade ainda não é notoriamente desenvolvida. Uma dessas regiões é o sul do estado do Pará. Esta região tem como sua principal atividade econômica a agropecuária, seguido de serviços e da indústria. Pensando no desenvolvimento da indústria local, o desenvolvimento das indústrias cerâmicas na região poderia contribuir com as receitas anuais, além da geração de emprego e renda na região [7].

Uma ocorrência de argila na zona rural na região do Araguaia, sul do Pará, despertou o interesse em estudar a possibilidade de uso deste recurso para produção de cerâmica vermelha. $\mathrm{O}$ trabalho teve como objetivo a avaliação de cenários técnicos e econômicos para a implantação de uma pequena unidade fabril de cerâmica, considerando esta região como um estudo de caso para prospecção de argila vermelha. 


\section{MATERIAIS E MÉTODOS}

\subsection{Sondagem da Área}

A execução das sondagens foi realizada em parceria com a GEOTEC Sondagens. A atividade atendeu ao disposto na norma técnica ABNT NBR 9603/86 - Sondagem a Trado e Termo de Referência [8] para a elaboração de diagnósticos, estudo de concepção, projetos básicos e executivos de engenharia, estudos ambientais e exploração de recursos minerais. As investigações diretas foram realizadas de modo a permitir a caracterização dos materiais da subsuperfície e retirada de amostras para caracterização.

Amostras da argila foram coletadas na zona rural da cidade de Santana do Araguaia - PA e encaminhadas ao Laboratório de Caracterização de Materiais do Instituto SENAI de Inovação em Tecnologias Minerais, em Belém - PA.

\subsection{Caracterização dos Materiais}

Análise de Umidade: os procedimentos para análise de umidade consistiram no emprego de vidros relógios como recipientes para as amostras. Os materiais foram mantidos em estufa a $105^{\circ} \mathrm{C}$ por $24 \mathrm{~h}$. Em seguida, as amostras foram pesadas em balança analítica.

Análise Mineralógica: as amostras foram secas a $105^{\circ} \mathrm{C}$ por $24 \mathrm{~h}$, cominuídas em moinho de panela modelo HSM $100 \mathrm{H}$, da Herzog, classificada em peneira manual de 100 mesh e homogeneizada. Análises mineralógicas por Difratometria de Raios-X (DRX) foram realizadas em equipamento modelo Empyrean Série 2, tubo de Raios-X cerâmico de Cobre e nível de potência máxima de 2,2 kW, da marca PANalytical. Os dados foram adquiridos através do software Data Collector e a interpretação foi realizada com software HighScore Plus. As condições de análise foram: fenda soller de $0,02 \mathrm{rad}$, filtro $\mathrm{k} \beta$ de $\mathrm{Ni}$, fenda divergente $1 / 4$, fenda antiespalhamento $1 / 2$, spinner 1 rps, detector $3 d$ PIXcel 1x1, ângulos de 3,5 a $75^{\circ} 2 \theta$, tamanho do passo 0,02 , tempo por passo $55 \mathrm{~s}, 40 \mathrm{kV}$ e $40 \mathrm{~mA}$.

Análise Química: amostras foram prensadas com proporção de 1 para 4 (Aglomerante WAX + Amostra). Análises químicas por Espectroscopia de Fluorescência de Raios-X por Energia Dispersiva (EDXRF) foram realizadas em equipamento modelo Epsilon $3^{\mathrm{XLE}}$, tubo de Raios-X cerâmico de Ródio e potência máxima de $15 \mathrm{~W}$, da marca PANalytical. Os dados foram adquiridos através do software Epsilon 3 e a interpretação foi realizada com a aplicação Omnian. Também foi realizada perda ao fogo (PF) com $1 \mathrm{~g}$ de amostra em mufla a $1.000^{\circ} \mathrm{C}$ por $1 \mathrm{~h}$.

\subsection{Análise de Cenários Técnicos e Econômicos}

A análise de cenários técnicos e econômicos foi baseada em informações de mercado e dados técnicos, não sendo estudadas informações específicas da empresa para geração de um cenário individual. Aspectos considerados nas análises: 1) avaliação técnica: estágio de desenvolvimento da tecnologia; competências técnicas; plataforma; complexidade e outros; 2) avaliação econômica: otimista; projetado; pessimista; e 3) construção dos cenários: emprego dos índices VPL - Valor Presente Líquido; TIR - Taxa Interna de Retorno; Payback - Retorno do Investimento.

\section{RESULTADOS E DISCUSSÃO}




\subsection{Estimativa da Capacidade de Extração e Duração da Jazida}

A sondagem a trado ST revelou uma incidência de Argila Vermelha com profundidade média de 0,4 m. A cubagem média do volume estimado para a Argila Vermelha foi de $30.000 \mathrm{~m}^{3}$.

A capacidade de extração e a duração da jazida para uma micro e pequena empresa foram estimadas com base no setor cerâmico do norte do estado do Pará [9]. Em uma suposta empresa de porte intermediário (Médio Porte), o consumo e a produção foram estimados através de interpolação dos dados entre uma micro e pequena empresa, como apresentado na Tabela 2, a qual apresenta também, a produção de peças.

Tabela 2. Estimativa de duração da argila conforme o porte de empresa.

\begin{tabular}{lccc}
\hline \multicolumn{1}{c}{ Tipo } & Consumo (ton/h) & $\begin{array}{c}\text { Produção } \\
\text { (mil peças/mês) }\end{array}$ & Duração (anos) \\
\hline Microempresa & 1,1 a 1,4 & $<100$ & $\sim 16$ \\
\hline Médio Porte & $\sim 2,7$ & 80 a 160 & $\sim 8,3$ \\
\hline Pequena Empresa & 5,5 a 6,5 & 100 a 300 & $\sim 3,7$ \\
\hline
\end{tabular}

Considerando o volume estimado de argila vermelha e a produção nominal de uma microempresa (até 100 mil peças/mês), o período de exploração da jazida poderia ser de cerca de 16 anos. Para uma pequena empresa, a qual a produção anual média é de até $300 \mathrm{mil} \mathrm{peças/mês,} \mathrm{estima-se} \mathrm{um} \mathrm{período} \mathrm{de} \mathrm{cerca} \mathrm{de} 4$ anos. Ressalta-se que durante o processo de produção, existem perdas decorrentes do manuseio, tais como, matéria-prima e produto final. Além disso, deve-se considerar o investimento inicial, a demanda de mercado local e os custos de operação envolvidos.

\subsection{Caracterização da Argila}

\subsubsection{Análise de Umidade}

Os resultados da análise de umidade são apresentados na Tabela 3 . O teor de umidade para as três alíquotas foi igual, o que infere uma umidade uniforme na argila.

Tabela 3. Teor de umidade, em triplicata, da Argila Vermelha.

\begin{tabular}{cccccc}
\hline \multirow{2}{*}{ Amostra } & \multicolumn{4}{c}{ Massa (g) } & \multirow{2}{*}{ Umidade } \\
\cline { 2 - 5 } & VR $^{*}$ & Amostra & Conj. & Conj.-VR & (\%) \\
\hline 1 & 42,90 & 5,01 & 47,34 & 4,44 & 11,40 \\
\hline 2 & 43,50 & 5,02 & 47,50 & 4,45 & 11,40 \\
\hline 3 & 42,86 & 5,01 & 47,30 & 4,44 & 11,40 \\
\hline
\end{tabular}

(*): Vidro Relógio (VR)

\subsubsection{Composição Mineralógica}

O difratograma da Argila Vermelha está apresentado na Figura 1. A amostra apresentou composição mineralógica composta por: argilomineral caulinita $(7,20 \mathrm{e}$ $3,52 \AA)$ e os minerais gibbsita $(4,84 \AA)$, quartzo $(3,34$ e $4,25 \AA)$ e anatásio $(3,52 \AA)$. 

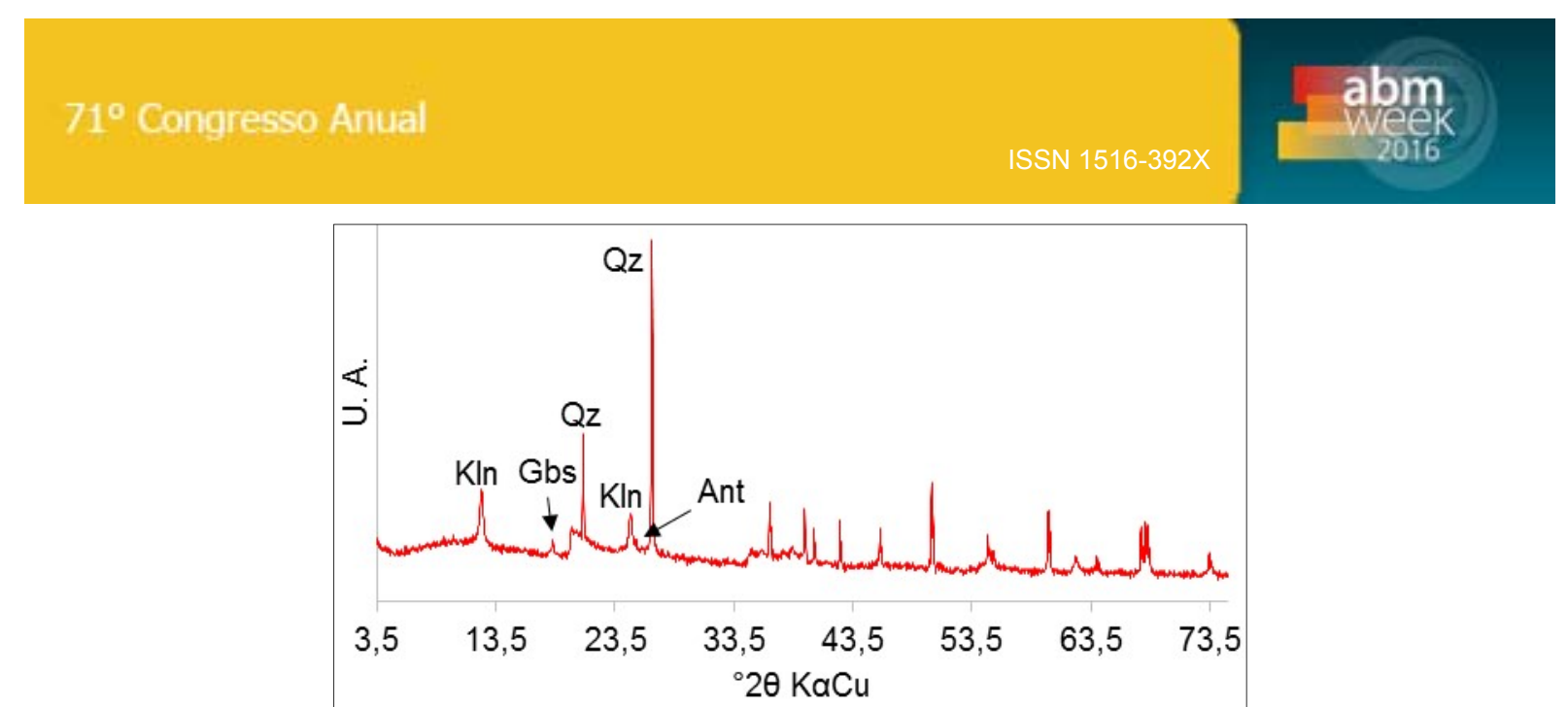

Figura 1. Difratograma da argila: Caulinita (KIn), Gibbsita (Gbs), Quartzo (Qz) e anatásio (Ant).

Estudos das propriedades de argilas para uso como cerâmica vermelha foram realizados [10, 11]. Macedo et al. [10] citam Grim [12], afirmando que o limite de plasticidade dos argilominerais crescem na ordem: caulinita bem cristalizada, caulinita mal cristalizada, illita e montmorillonita. Considerando esta afirmação, por conter apenas o argilomineral caulinita, a amostra de Argila Vermelha poderá apresentar plasticidade reduzida, levando a uma possibilidade da demanda de realizar uma mistura com argilas que apresentem maior volume de argilominerais e, portanto, apresentando maior plasticidade.

\subsubsection{Composição Química}

O resultado da análise química da amostra é apresentado na Tabela 4.0 silício foi o elemento predominante na amostra $(55,55 \%)$, sendo a sua presença associada aos minerais quartzo e caulinita. O segundo elemento em maior concentração, o alumínio $(32,55 \%)$, possui associação aos minerais caulinita e gibbsita. O Ferro, terceiro elemento mais abundante $(2,32 \%)$, é associado ao mineral Hematita (apesar deste não ter sido encontrado), devido à coloração avermelhada da argila. O titânio é associado ao anatásio, e o zircônio não foi associado aos minerais identificados. A Perda ao Fogo (PF), referente a materiais voláteis, como água adsorvida e estrutural, matéria orgânica, carbonatos, etc. [13], e apresentou valor de 8,20\%.

Tabela 4. Composição Química da Argila Vermelha.

\begin{tabular}{cc}
\hline Elemento & Concentração (\%) \\
\hline $\mathrm{SiO}_{2}$ & 55,55 \\
\hline $\mathrm{TiO}_{2}$ & 1,10 \\
\hline $\mathrm{ZrO}_{2}$ & 0,10 \\
\hline $\mathrm{Al}_{2} \mathrm{O}_{3}$ & 32,55 \\
\hline $\mathrm{Fe}_{2} \mathrm{O}_{3}$ & 2,32 \\
\hline $\mathrm{PF}$ & 8,20 \\
\hline Outros $^{*}$ & 0,18 \\
\hline
\end{tabular}

$\left(^{*}\right)$ : Somatório dos elementos com concentração inferior a $0,1 \%$.

\subsection{Análise de Cenários Técnicos e Econômicos}

Atualmente, a região de Santana do Araguaia possui empresas ceramistas. Em regiões próximas, também, podem ser encontradas unidades fabris em Conceição do Araguaia (PA) e Vila Rica (MT).

Considerando que o PIB da região de Santana do Araguaia, em 2013, foi de aproximadamente $\mathrm{R} \$ 316$ milhões, sendo $43 \%$ pela agropecuária, $34 \%$ por serviços 
e $23 \%$ pela indústria, isto pode representar um potencial mercado para a cerâmica e desenvolvimento de novos empreendimentos da cidade e sua região, como obra de construção de residências, mercados, infraestrutura urbanas e rurais, etc.

Para a implantação de uma unidade fabril de cerâmica, faz-se necessário considerar o investimento inicial com a aquisição dos equipamentos, o transporte destes e dos materiais de construção para galpões, fornos etc., bem como para a construção da base para a unidade fabril. Uma fábrica de tijolos, basicamente, precisa de área para armazenamento de argila, área de extrusão (sala de máquinas), estufa para secagem dos produtos e fornos para a queima.

Uma análise com base em um negócio intermediário entre uma micro e pequena empresa produtora de cerâmica foi realizada considerando produção estimada de tijolos de 06 (seis) furos $(09 \times 14 \times 19 \mathrm{~cm})$. No local estudado já se tem área suficiente para instalação e posterior ampliação do negócio proposto, além de um volume de argila estimado em pelo menos $30.000 \mathrm{~m}^{3}$, conforme relato da análise de cubagem. A necessidade da adição de uma argila com características diferentes da estudada poderia ser demandada, conforme necessidade do produto.

\subsubsection{Estimativa de Cenários para uma Fábrica de Tijolo Cerâmico}

A proposta de negócio considera a empresa atendendo ao mercado local, considerando que as empresas ceramistas da região estão distantes, deixando espaço para o desenvolvimento desta nova unidade fabril. A empresa, por ser de um porte intermediário, a priori, poderia atender a uma demanda de 140 milheiros de tijolos por mês, com dois fornos do tipo paulistinha, com capacidade de produzir até 20 milheiros de tijolos de 06 furos $(09 \times 14 \times 19 \mathrm{~cm})$, em cada fornada, em um ciclo de 4 dias. Estimativas baseadas em valores de mercado apontam que o milheiro deste produto é comercializado com valores entre $R \$ 230,00$ e $R \$ 430,00$ [9].

Foram considerados a taxa de depreciação dos equipamentos, galpões, fornos e instalações, em $8,0 \%$ a.a. O encargo social considerado foi para uma pequena empresa optante pelo SIMPLES NACIONAL [14] e um reajuste salarial anual de aproximadamente $8,3 \%$, baseado na média inflacionária dos últimos 4 anos, contando inicialmente com 6 operários e gerenciado sem custos pelo proprietário da empresa. Considerando a retração volumétrica após a queima, de 6 a $10 \%$, e perdas que ocorrem durante o processamento $[15,16]$, para cada $\mathrm{m}^{3}$ de argila podem ser fabricados cerca de 874 tijolos. O valor considerado para o milheiro foi de $R \$ 250,00$, próximo ao mínimo praticado e assim dando margem de aumento no valor do produto. Neste sentido, as estimativas levam em consideração a produção final com um lucro bruto mensal de pelo menos $\mathrm{R} \$ 35.000,00$.

O investimento inicial, calculados com base em trabalhos $[17,18]$, ficam em torno de $\mathrm{R} \$ 290.000,00$ para a construção dos galpões em estruturas metálicas e os fornos, e cerca de $\mathrm{R} \$ 263.000,00$ para aquisição dos equipamentos.

Para a estimativa dos cenários econômicos, o Valor Presente Líquido (VPL), a Taxa Interna de Retorno (TIR) e o Payback, foi considerado investimento inicial de $R \$ 553.000,00$, receita bruta de $R \$ 35.000,00$ e principais custos variáveis de 09 a $12 \%$. Assim, três cenários foram considerados: $1^{\circ}$ ) projetado, considerando o milheiro comercializado a $\mathrm{R} \$ 250,00 ; 2^{\circ}$ ) otimista, considerando a venda do milheiro a $\mathrm{R} \$ 275,00 ;$ e $3^{\circ}$ ) pessimista, o valor do milheiro a $\mathrm{R} \$ 237,50$ e aumento no custo total em $3,0 \%$ a.a.

Para os três cenários foi suposto que haveria o financiamento de $50 \%$ do investimento inicial a uma taxa de aproximadamente $11 \%$ a.a. A taxa de desconto atual é uma porcentagem que abate o valor da VPL, a qual considera as taxas de investimento 
mais seguro (financiamento), a inflação e o capital financiado. Os resultados para o VPL, TIR e para o Payback são mostrados na Tabela 5.

Nota-se que para o cenário projetado os valores de VPL foram positivos e a TIR foi cerca de $5 \%$ superior à taxa de desconto atual. Este cenário mostra que o investimento é recuperado em torno de 47 meses comercializando o milheiro do tijolo ao valor de $\mathrm{R} \$ 250,00$. Para o cenário otimista, o VPL mostrou-se positivo e a TIR foi cerca de $31 \%$, ou seja, $15 \%$ superior à taxa de desconto atual e o investimento teria retorno em 40 meses. Considerando o cenário pessimista, com custos aumentando em $3 \%$ a.a. e milheiro comercializado a $R \$ 237,50$, o projeto apresenta uma baixa perspectiva de retorno.

Tabela 5. Estimativas para os Cenários Econômicos

\begin{tabular}{lccc}
\hline \multicolumn{1}{c}{ Indicador } & $\mathbf{1}^{\mathbf{0}}$ Cenário & $\mathbf{2}^{\mathbf{0}}$ Cenário & $\mathbf{3}^{\mathbf{0}}$ Cenário \\
\hline Investimento inicial $(\mathrm{R} \$)$ & $553.000,00$ & $553.000,00$ & $553.000,00$ \\
\hline Taxa de desconto atual $(\%)$ & 16 & 16 & 16 \\
\hline VPL $(\mathrm{R} \$)$ & $55.787,04$ & $191.913,94$ & $-26.359,27$ \\
\hline TIR $(\%)$ & 21 & 31 & 14 \\
\hline Payback (meses) & 47 & 40 & 54 \\
\hline
\end{tabular}

\section{CONCLUSÃO}

A sondagem, com base nos parâmetros estabelecidos, revelou uma cubagem de reserva mineral de aproximadamente $30.000 \mathrm{~m}^{3}$ de argila vermelha. Considerando estimativas baseadas em mercado e literatura, a análise de cenários levou a uma possibilidade de implementação de uma unidade fabril de cerâmica com capacidade produtiva de até 140 mil peças/mês, comercializada no valor mínimo do milheiro de $\mathrm{R} \$ 250,00$. Ressalta-se restrições quanto ao tipo de produto desejado e a característica da argila prospectada, podendo ser necessário a mistura de argilas com maior quantidade de argilominerais.

\section{REFERÊNCIAS}

1 Guggenheim S, Hillier S, Jaynes W, Maurice P, Pozo M, Velbel M. The Clay Minerals Society Glossary for Clay Science Project. The Clay Minerals Society. 2015 [acesso em 16 mar. 2016]. Disponível em:

http://www.clays.org/GLOSSARY/ClayTermsApril2015.pdf.

2 BRASIL. Anuário Mineral Brasileiro. Departamento Nacional de Produção Mineral. 2010 [acesso em: 28 mar. 2016]; 35. Disponível em: http://www.dnpm.gov.br/assuntos/aopublico/anuario-mineral/arquivos/ANUARIO_MINERAL_2010.pdf.

3 Bustamante GM, Bressiani JC. A Indústria Cerâmica Brasileira. Cerâmica Industrial. 2000;5(3): 31-36.

4 Junior MC, Tanno LC, Sintoni A, Motta JFM, Coelho JM. A Indústria de Cerâmica Vermelha e o Suprimento Mineral no Brasil: Desafios para o Aprimoramento da Competitividade. Cerâmica Industrial. 2012;17(1): 36-42.

5 Prado US, Bressiani JC. Panorama da Indústria Cerâmica Brasileira na Última Década. Cerâmica Industrial. 2013;18(1): 7-11.

6 Santos CVP, Silva AR, Filho MASG, Neto EF, Rabelo AA. Índice de Plasticidade e Análise Racional de Argilas de Marabá (PA) para Avaliação das Zonas de Extrusão. Cerâmica Industrial. 2012;17(2): 25-27.

7 Instituto Brasileiro de Geografia e Estatística [página da internet]. Rio de Janeiro: IBGE, 2016 [acesso em 20 jan. 2016]. Disponível em: http://cidades.ibge.gov.br/xtras/te- 
mas.php?lang=\&codmun=150670\&idtema=152\&search=para|santana-do-

araguaia|produto-interno-bruto-dos-municipios-2013.

8 Associação Brasileira de Normas Técnicas. NBR 9603: Sondagem a Trado. Rio de Janeiro, 1986. 6 p.

9 Instituto Nacional de Tecnologia. Avaliação do Setor de Ceramica da Regiao Norte do Estado do Pará - Regioes de Abaetetuba, Igarape-Miri e São Miguel do Guama. 2011. Programa de Eficiencia Energética en Ladrilleras Artesanales de America Latina para Mitigar el Cambio Climaticos. 12 p.

10 Macedo RS, Menezes RR, Neves GA, Ferreira HC. Estudo de Argilas Usadas em Cerâmica Vermelha. Cerâmica. 2008;54: 411-417.

11 Junior LAG, Varajão AFDC, Souza MHO, Moreno MMT. Estudo Comparativo das Olarias dos Municípios de Alfenas e Areado, MG. 2012;58: 404-411.

12 Grim RE. Clay Mineralogy. New York: McGraw-Hill Book; 1968.

13 Santos PS. Tecnologias de Argilas. São Paulo: Edgard Blücher;1975.

14 BRASIL. Simples - Microempresa (ME) e Empresa de Pequeno Porte (EPP). Ministério da Fazenda. 2005 [acesso em: 20 mar. 2016]. Disponível em:

http://www.receita.fazenda.gov.br/PessoaJuridica/DIPJ/2005/PergResp2005/pr108a200. htm

15 Batista VR, Nascimento JJS, Lima AGB. Secagem e Queima de Tijolos Cerâmicos Maciços e Vazados Incluindo Variações Dimensionais e Danos Estruturais. 2008;3(1): 46-61.

16 Shinomiya, LD, Gomes, JO, Alves, JO. Planejamento de cenários para uso de resíduos industriais: aplicação para lama vermelha. Revista Gestão em Engenharia, 2015;2: 4366.

17 Silva AC. Análise de Viabilidade Econômica Financeira para a Implantação de uma Central de Massa em uma Indústria Cerâmica de Itaboraí, RJ [dissertação de mestrado]. Rio de Janeiro: Centro Federal de Educação Tecnológica Celso Suckow da Fonseca; 2012.

18 Serviço Brasileiro de Apoio às Micro e Pequenas Empresas. Fornos do Setor de Cerâmica Vermelha do Rio Grande do Norte. 2014. Relatório de Assessoria Técnica e Tecnológica. $20 \mathrm{p}$. 